In Heft 3/95, S. 3036, hat JurPC unter dem Titel "Müssen wir neu schreiben lernen? - Die Rechtschreibreform aufieinen Blick" einen Überblick über die zu erwartende Neuregelung der deutschen Rechtschreibung abgedruckt. Autor war Dr. Klaus Heller (Institut für deutsebe Sprache, Mannheim), der die Bemühungen um die Reform der deutschen Orthographie koordiniert. Er hatte im November 1994 an den dritten Wiener Gesprächen zur Neuregelung der deutschen Rechtschreibung teilgenommen und den dort erreichten Stand wiedergegeben.

Im:folgenden berichtet Klaus Heller über die Entwicklung nach der Wiener Konferenz und vermittelt den aktuellen Stand der Reform, die nun bald Wirklichkeit werden soll.

\title{
Rechtschreibreform
}

\section{Klaus Heller}

\section{Was geschah seit der Wiener Konferenz?}

Nachdem eine in Wien benannte international besetzte Redaktionsgruppe, der auch Fachbeamte angehörten, die Beschlüsse von Wien in das Regelwerk eingearbeitet hatte und das Wörterverzeichnis fertiggestellt worden war, konnte am 13. April 1995 die vollständige Vorlage für das amtliche Regelwerk den zuständigen Behörden in Deutschland, in Österreich und in der Schweiz übergeben werden. Um jedem Interessierten Einblick in das vollständige Regelwerk zu ermöglichen und besonders allen, die sich - wie viele Verlage - auf die neue Rechtschreibung einstellen mußten, eine längere Vorbereitungszeit zu geben, veröffentlichte der Internationale Arbeitskreis für Orthographie Anfang Juli 1995 die vollständige Vorlage für das amtliche Regelwerk im Gunter Narr Verlag, Tübingen.

Dem großen Informationsbedürfnis der Bevölkerung entgegenkommend, erschienen nach der ersten Auflage der Sprachreport-Extraausgabe ${ }^{1}$ und der Broschüre des Duden-Verlags ${ }^{2}$ (beide noch im Dezember 1994) zahlreiche Nachdrucke dieser Ausgaben sowie weitere Publikationen von Ebner/Fussy ${ }^{3}$, Zabel $^{4}$ und Heller ${ }^{5}$.

Während die Regierungen Österreichs und der Schweiz dem Neuregelungsvorschlag umgehend zustimmten, erhoben einzelne deutsche Politiker Einspruch gegen einige veränderte Wortschreibungen ${ }^{6}$. Erneut erhoben sich zum Teil heftige Debatten. Immer wiederkehrende Fehlinformationen in Presse, Funk und Fernsehen veranlaßten das Institut für deutsche Sprache, im November 1995 eine Presseerklärung herauszugeben, die der Richtigstellung von Falschmeldungen diente. Ende September 1995 beauftragte die Kultusministerkonferenz eine Kommission ("länderoffene Amtschefskommission") damit, noch bestehende Probleme für eine abschließende Beratung und Entscheidung aufzubereiten. Diese Kommission hatte außerdem die Beschlüsse der Ministerpräsidentenkonferenz vom 26./27. Oktober $1995 \mathrm{zu}$ berücksichtigen, die auch auf längere Übergangsfristen orientierten. Im Ergebnis dieser Beratungen wurden einige veränderte Wortschreibungen zurückgenommen und eine Verlängerung der vorgesehenen Fristen vorgeschlagen. Diese Änderungen wurden daraufhin mit Österreich und mit der Schweiz abgestimmt. Auf ihrer Plenarsitzung am 30. November 1995 und 1. Dezember 1995 stimmten schließlich die Kultusminister Deutschlands der Neuregelung der deutschen Rechtschreibung zu.

Dieser Beschluß wurde am 14. Dezember von der Konferenz der Ministerpräsidenten gebilligt. Am 17. April fand eine Unterrichtung des Bundeskabinetts statt. Damit steht der

\footnotetext{
1 Heller, Klaus: Rechtschreibreform. Die Reform auf einen Blick. SPRACHREPORT-Extraausgabe, Dezember 1994. Institut für deutsche Sprache, Mannheim.

2 Sitta, Horst/Gallmann, Peter: Informationen zur neuen deutschen Rechtschreibung. Duden-Verlag, Mannheim/Leipzig/Wien/Zürich 1994.

3 Ebner, Jakob/Fussy, Herbert: Rechtschreibung neu. Eine Einführung in die neue Ortografie. ÖBV Pädagogischer Verlag, Wien 1995.

4 Zabel, Hermann: Die neue deutsche Rechtschreibung. Falken-Verlag, Niedernhausen/Ts. 1995.

5 Heller, Klaus: Rechtschreibung 2000. Die Reform auf einen Blick. Wörterliste der geänderten Schreibungen. Ernst Klett Schulbuchverlag, Stuttgart/Düsseldorf/Berlin/Leipzig 1995.

6 Vgl. das SPIEGEL-Interview mit dem bayerischen Kultusminister Hans Zehetmair (SPIEGEL 37/1995).
}

\section{April 95:}

Übergabe des vollständigen amtlichen Regelwerks

Einspruch aus Deutschland

Dr. Klaus Heller ist Mitarbeiter de Instituts für deutsche Sprache in

Mannheim, Mitglied der Kommission für Rechtschreibfragen am IDS und Mitglied des Internationalen Arbeitskreises für Orthographie.

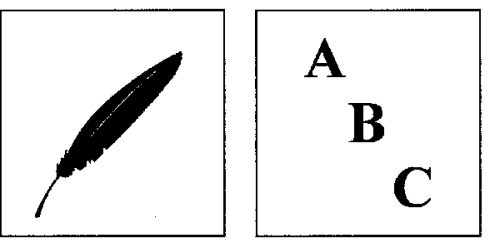

\begin{tabular}{l} 
Dr. Klaus Heller ist Mitarbeiter des \\
Instituts für deutsche Sprache in \\
Mannheim, Mitglied der Kommission \\
für Rechtschreibfragen am IDS und \\
Mitglied des Internationalen Arbeits- \\
kreises für Orthographie. \\
\hline
\end{tabular}




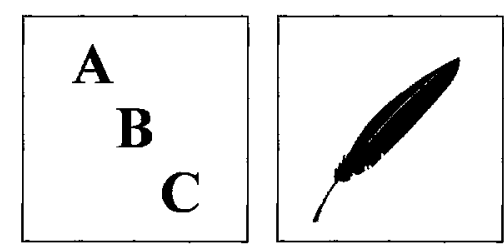

Die Änderungen der Änderungen

1.'Juli 1996:

Unterzeichnung der gemeinsamen Erklärung

1. August 1998: Einfübrung in den Schulen baldigen Unterzeichnung einer Gemeinsamen Erklärung der deutschsprachigen Staaten zur Einführung der Reform nichts mehr im Wege.

\section{Welche inhaltlichen Änderungen haben sich seit der Wiener Konferenz vom November 1994 ergeben?}

Auf den Beratungen der Amtschefskommission, an denen auch drei Sprachwissenschaftler des Internationalen Arbeitskreises für Orthographie teilnehmen konnten, wurde an den neuen Regeln keine Kritik geübt. Änderungsvorschläge zielten ausschließlich auf die $\mathrm{Zu}$ rücknahme einzelner in Wien bereits beschlossener Reformschreibungen, wodurch zum Teil Ausnahmen weiter bestehen bleiben. Zusammengefaßt stellen sich diese "Änderungen der Änderungen" wie folgt dar:

- Nicht gebilligt wurden Karrosse/Karrosserie, Packet, Pott/Jackpott und Zigarrette/Zigarrillo.

- Bonbonniere, Chansonnier, Saisonnier und Ordonnanz bleiben die Hauptformen (daneben Bonboniere, Chansonier, Saisonier und Ordonanz).

- Schreibungen mit diakritischen Zeichen (z. B. Château) stehen gleichberechtigt neben Schreibungen ohne diakritische Zeichen (z. B. Chateau).

- Kommuniqué bleibt Hauptform (daneben Kommunikee).

- Nicht gebilligt wird Restorant.

- Nicht gebilligt wird Holokaust.

- Außer (wie vorgeschlagen) bei phon/phot/graph wird $f$ statt $p h$ bei Delfin akzeptiert. Die Hauptform bleibt Delphin.

- Nicht gebilligt wurden Rabarber, Reuma und Rytmus (einschließlich Eurytmie). Eurythmie ist als Nebenform zugelassen. Gebilligt sind Katarr, Myrre und Hämorriden als Nebenformen.

- Die $t$-Schreibung anstelle der $t h$-Schreibung ist lediglich bei Panter und bei Tunfisch möglich. Die Hauptformen sind Panther und Thunfisch. Alle anderen th-Schreibungen bleiben erhalten.

- Die Schreibungen Frevel/freventlich, Febde und Thron werden nicht geändert.

- Die Schreibungen Albtraum und Alptraum stehen gleichberechtigt nebeneinander (keine semantische Differenzierung).

- Großschreibung gilt für Heiliger Vater, Letzte Ölung und Dritte Welt. Differenziert wird nach Eigennamen und Begriff bei Eiserner/eiserner Vorbang und Kalter/kalter Krieg.

Nachdem diese Korrekturen sowohl von der Kultusministerkonferenz als auch von der Konferenz der Ministerpräsidenten bestätigt worden waren und auch Einvernehmen mit den zuständigen Stellen Österreichs und der Schweiz hergestellt war, konnte das Regelwerk am Institut für deutsche Sprache einer entsprechenden Überarbeitung unterzogen werden. Es wurde in dieser, nun endgültigen Form die Grundlage für die Gemeinsame Erklärung, die am 1. Juli 1996 von Deutschland, Österreich und der Schweiz sowie weiteren interessierten Staaten in Wien unterzeichnet worden ist.

\section{Wann wird die neue Regelung in Kraft treten?}

Zunächst war vorgesehen, die neue Rechtschreibung bereits 1997 in die Schulen einzuführen. Im Ergebnis der politischen Beratungen in Deutschland ist dieser Termin inzwischen korrigiert worden. Nun soll erst mit Beginn des Schuljahrs 1998/99 (1. August 1998) in den Schulen ausschließlich die neue Rechtschreibung gelehrt werden. Auch die Übergangszeit ist verlängert worden. Danach wird die bisherige Schreibung bis zum Ende des Schuljahres 2004/05 wohl als überholt, jedoch nicht als falsch gelten. Eine Übergangszeit, in der es möglich sein wird, Schüler und Schülerinnen bereits mit der neuen Rechtschreibung bekanntzumachen, soll es auch für das Schuljahr 1997/98 geben. Es darf davon ausgegangen werden, daß es außerschulischen Bereichen (Behörden, Verlagen, Presseorganen usw.) freisteht, nach Unterzeichnung der Gemeinsamen Erklärung in Wien die neue Rechtschreibung zu praktizieren. 


\section{Wer wird die neue Rechtschreibung "verwalten"?}

Es bleibt dabei, daß eine Zwischenstaatliche Kommission für die deutsche Rechtschreibung hinfort die Entwicklung der deutschen Rechtschreibung beobachten und gegebenenfalls den staatlichen Stellen Korrekturen oder Ergänzungen vorschlagen wird, die dann von den Wörterbuchredaktionen umzusetzen wären. Die Kommission, die ihren Sitz am Institut für deutsche Sprache in Mannheim haben wird, hat darüber zu wachen, daß die amtlichen Regeln nicht willkürlich ausgelegt oder verändert werden. Sie wird dabei mit Praktikern (Pädagogen, Lexikographen usw.) und Fachbeamten zusammenarbeiten und den staatlichen Stellen über ihre Arbeit regelmäßig Rechenschaft ablegen.

\section{Literatur (in Auswahl)}

Deutsche Rechtschreibung. Regeln und Wörterverzeichnis.

Vorlage für die amtliche Regelung.

Herausgegeben vom Internationalen Arbeitskreis für Orthographie.

Gunter Narr Verlag, Tübingen 1995 (aktuelle Korrekturliste beigefügt).

\section{Rechtschreibreform.}

Eine Zusammenfassung von Dr. Klaus Heller.

SPRACHREPORT-Extraausgabe, Januar 1996.

Institut für deutsche Sprache, Mannheim.

Die SPRACHREPORT Extraausgabe Januar 1996 sowie weitere aktuelle Informationen zur Rechtschreibreform können auch über das Internet abgerufen werden:

http://www.ids-mannheim.de

\section{Rechtschreibung 2000.}

Die aktuelle Reform. Wörterliste der geänderten Schreibungen.

2., aktualisierte und erweiterte Auflage.

Ernst Klett Schulbuchverlag Stuttgart/Düsseldorf/Berlin/Leipzig 1996.

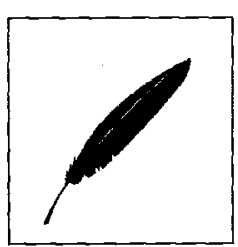

A

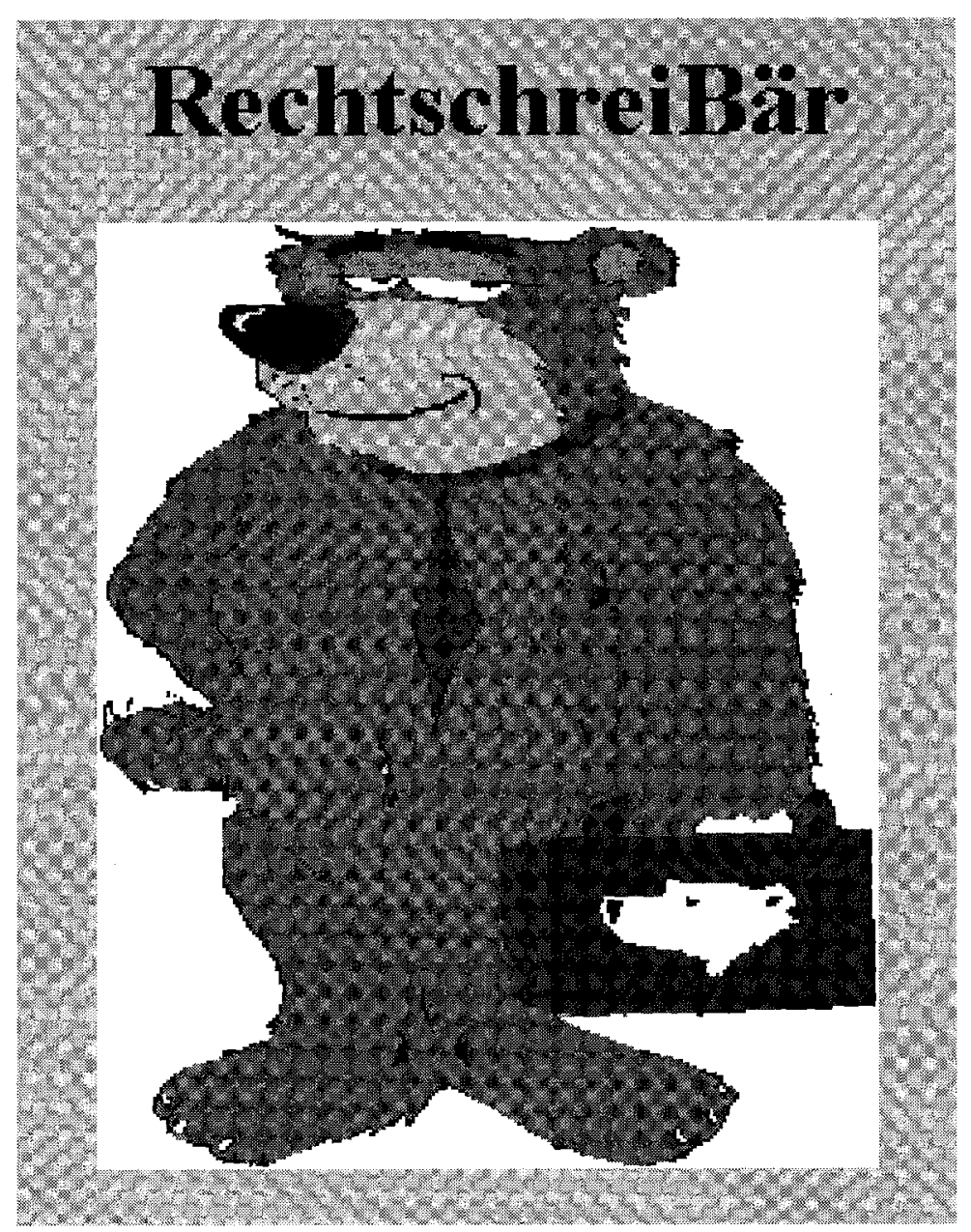

Quelle:

bttp://rererwids-mannheim.de/pub/baer.html 